\title{
Ataxia telangiectasia mutated (ATM) interacts with p400 ATPase for an efficient DNA damage response
}

\author{
Rebecca J. Smith, Matthew S. Savoian, Lauren E. Weber and Jeong Hyeon Park
}

\begin{abstract}
Background: Ataxia telangiectasia mutated (ATM) and TRRAP proteins belong to the phosphatidylinositol 3-kinaserelated kinase family and are involved in DNA damage repair and chromatin remodeling. ATM is a checkpoint kinase that is recruited to sites of DNA double-strand breaks where it phosphorylates a diverse range of proteins that are part of the chromatin and DNA repair machinery. As an integral subunit of the TRRAP-TIP60 complexes, P400 ATPase is a chromatin remodeler that is also targeted to DNA double-strand break sites. While it is understood that DNA binding transcriptional activators recruit p400 ATPase into a regulatory region of the promoter, how p400 recognises and moves to DNA double-strand break sites is far less clear. Here we investigate a possibility whether ATM serves as a shuttle to deliver p400 to break sites.

Results: Our data indicate that p400 co-immunoprecipitates with ATM independently of DNA damage state and that the N-terminal domain of p400 is vital for this interaction. Heterologous expression studies using Sf9 cells revealed that the ATM-p400 complex can be reconstituted without other mammalian bridging proteins. Overexpression of ATM-interacting p400 regions in U2OS cells induced dominant negative effects including the inhibition of both DNA damage repair and cell proliferation. Consistent with the dominant negative effect, the stable expression of an $\mathrm{N}$-terminal p400 fragment showed a decrease in the association of p400 with ATM, but did not alter the association of p400 with TRRAP.
\end{abstract}

Conclusion: Taken together, our findings suggest that a protein-protein interaction between ATM and p400 ATPase occurs independently of DNA damage and contributes to efficient DNA damage response and repair.

Keywords: Phosphatidylinositol 3-kinase, Chromatin remodelling, DNA damage response, DNA repair, p400, ATM

\section{Background}

Ataxia telangiectasia mutated (ATM) and TRRAP belong to the phosphoinositide 3-kinase-related protein kinase (PIKK) family and play a critical role in recognising a DNA double-strand break (DSB) site as well as in remodeling chromatin to facilitate DNA repair processes [1-4]. The kinase substrates of ATM include a wide variety of proteins that are critically required for DNA repair and check point control [5-9]. In addition to orchestrating the activity of DNA repair machineries, ATM can control chromatin structure indirectly through protein

*Correspondence: j.park@massey.ac.nz

Institute of Fundamental Sciences, Massey University, Palmerston North, New Zealand phosphorylation [10]. In its arguably most important function, activated ATM induces nuclear foci around the DSB site involving phosphorylation of H2A.X at serine 139 ( $\gamma$-H2A.X) [3]. Histone $\gamma$-H2A.X has been used as an indicator of the degree of DNA damage as well as the effectiveness of the DNA repair process [11].

Localised chromatin relaxation around DSBs appears to be an ATM-independent process in which TRRAP participates through recruiting p400 and TIP60 to break sites $[2,12,13]$. TRRAP forms multi-subunit TRRAPTIP60 complex (human NuA4 complex) containing the chromatin remodeler $\mathrm{p} 400$ and histone acetylase TIP60 as an integral subunit where TRRAP, p400 and TIP60 are all required for DNA damage-induced chromatin 
decondensation [14-16]. The mechanism of how p400 is targeted to the DSB is poorly understood but the interaction of p400-containing complexes with DNA repair proteins such as Mdc1 and Rad51 have been suggested as a recruitment mechanism [17]. Taken together, DSBs seem to induce chromatin relaxation in both ATMdependent and independent manners. Importantly, ATM and TRRAP appear to act independently for efficient DNA repair by targeting different enzyme activities to the DSB sites. ATM and TRRAP have a modular domain structure, with a common FRAP-ATM-TRRAP-C-terminal (FATC) domain that is interchangeable in terms of the interaction ability with TIP60 [18]. The swappable domain of PIKK proteins suggests that there may be additional binding partners shared between members of the PIKK family. Here we demonstrate that the p400 ATPase, which has previously been shown to interact with TRRAP $[19,20]$ associates with ATM independently of DNA damage, providing an additional mechanism of ATM-dependent chromatin relaxation and p400 targeting.

\section{Results \\ The p400 associates with ATM in HEK293T cells}

The conserved structural features of ATM and TRRAP suggest that they interact with various proteins in common for their specific functions in DNA damage response and transcriptional regulation [1]. To examine whether p400 associates with ATM, similar to its interaction with TRRAP, co-immunoprecipitation assays were performed using different epitope tagged ATM, p400 and TIP60 proteins (Fig. 1a). The association of TIP60 with either ATM or p400 has previously been described and was used here as positive controls (Fig. 1a, right panel, lanes 6 and 7) $[19,21]$. Despite its low expression relative to HATIP60, HA-p400 was also able to co-immunoprecipitate with Flag-ATM (Fig. 1c, right panel, lanes 7 and 8). This raises the possibility that $\mathrm{p} 400$ could be a novel interacting partner of ATM in addition to TIP60. The interaction studies using endogenous ATM and p400 have not been successful mainly due to the lack of immunoprecipitation grade antibodies and relatively low expression levels of these high molecular weight proteins. However, Flagp400 ectopically expressed in HEK293T cells was able to precipitate endogenous ATM (Fig. 1b). As the interaction of p400 with ATM occurs in the endogenous level of ATM, the interaction would be physiologically relevant.

To examine the possibility that the association of p400 with ATM occurs indirectly through the multi-subunit TRRAP-TIP60 complex, co-immunoprecipitation experiments were performed between ATM and two integral subunits of the TRRAP-TIP60 complex, BAF53 and GAS41 (Fig. 1c) [22, 23]. HEK293T cells were transfected with plasmids expressing Flag-ATM, HA-TIP60, HAGAS41 and HA-BAF53 in various combinations and the whole cell extract was subjected to immunoprecipitation using anti-HA antibody-conjugated beads. The input and immunoprecipitation samples were analysed by immunoblotting using anti-HA and anti-Flag antibodies. The result showed that HA-TIP60 immunoprecipitated with Flag-ATM as expected whereas the other two members of the TRRAP-TIP60 complex did not associate, even under a forced overexpression setting (Fig. 1c, lane 8 versus lanes 6,7$)$. This result would suggest that the interaction of p400 with ATM might occur independently from the previously identified nuclear complexes containing GAS41 or BAF53.

Both ATM and p400 are targeted to the site of DSBs and play a critical role in DNA repair [3, 12, 15, 24]. To investigate whether the interaction between ATM and p400 is dependent on DNA damage, HEK293T cells were transfected in duplicate with Flag-ATM alone or together with either HA-TIP60 or HA-p400 and the interaction was examined in the presence and absence of bleomycin, an inducer of DSBs (Fig. 1d). To obtain comparable co-expression levels of Flag-ATM and HA-p400 in HEK293T cells, higher molar ratio of plasmid DNA expressing $\mathrm{p} 400$ was used for the transient co-expression and thereby resulted in a less expression of Flag-ATM in cells (Fig. 1d, lanes 7, 8). As expected, bleomycin induced an increase in H2A.X phosphorylation but did not alter the expression levels of ATM, p400 or TIP60 within the duplicate of transfection (Fig. 1d, left panel). Consistent with a previous report, the levels of ATM associated with TIP60 were remained similar regardless of DNA damage (Fig. 1d, right panel, compare lanes 5, 6) [25]. Similarly, the level of ATM associated with p400 did not change significantly in the presence of DNA damage (Fig. 1d, right panel, compare lanes 7,8 ), suggesting that a minor portion of ATM is associated with p400 irrespective of DNA damage.

\section{The interaction between p400 and ATM can be reconstituted in insect cells}

The common recruitment of ATM and multiple p400-containing complexes to the site of DNA damage makes it technically challenging to characterise p400-containing nuclear complexes at the DSB sites. To overcome such ambiguous localisation of multi-subunit p400 complexes and exclude the possibility of bridging proteins for the ATM interaction, we employed a heterologous gene expression system in baculovirus-infected Sf9 cells (Fig. 2). HA-RAR is the HA-tagged nuclear retinoic acid receptor and was used as a negative control to confirm that the interaction observed between Flag-ATM and HA-p400 was not dependent on the HA tag or an 


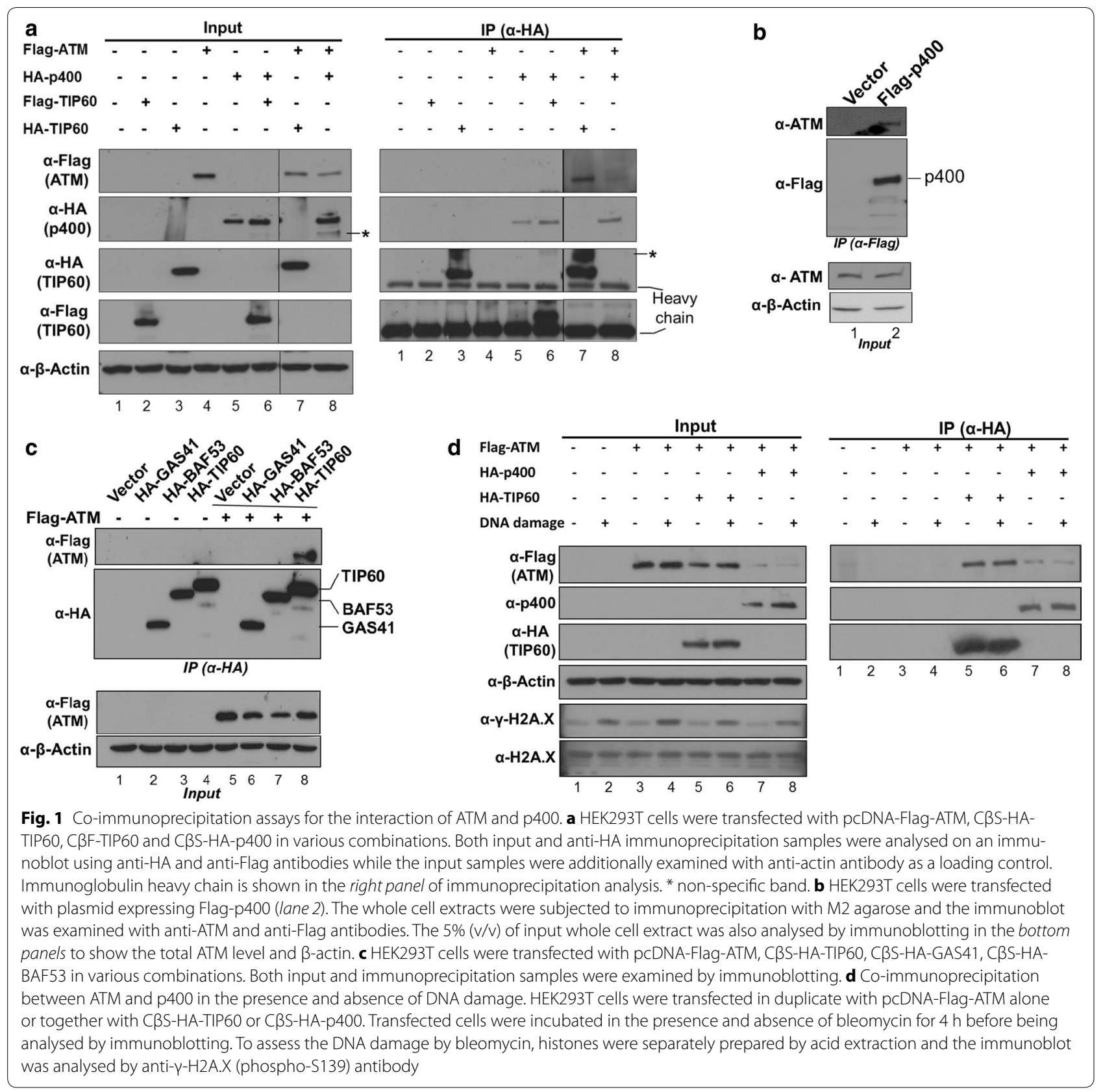

overexpression-derived artefact. The cells were harvested at $48 \mathrm{~h}$ post-infection to prepare whole cell extracts. Expression of recombinant proteins was confirmed by immunoblotting (Fig. 2a). HA-p400 was expressed at a low level with significant protein degradation or possibly truncated protein expression which was not improved by altering the time course of post infection. It is likely due to the large size and instability of the recombinant protein (Fig. 2a, lanes 3, 6). Therefore, higher viral titre of baculovirus expressing p400 was used to compensate a low expression level in which the co-expression level of Flag-ATM was reduced proportionally (Fig. 2a, lane 6 ). The whole cell extracts were divided into two equal volumes and subjected to immunoprecipitation using anti-HA antibody-conjugated beads (Fig. 2b) or antiFlag antibody-conjugated M2 agarose (Fig. 2c). Figure 2b shows that HA-p400 and HA-TIP60 co-immunoprecipitated with Flag-ATM respectively, while significantly overexpressed HA-RAR did not. Given that HA-p400 is expressed at markedly lower levels than HA-TIP60, the association of p400 with ATM appears more efficient than that of TIP60 in Sf9 cells (Fig. 2b, compare 


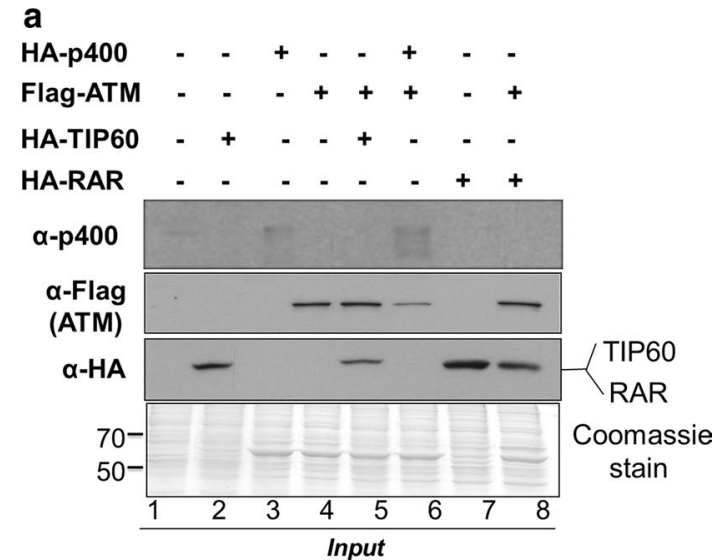

b

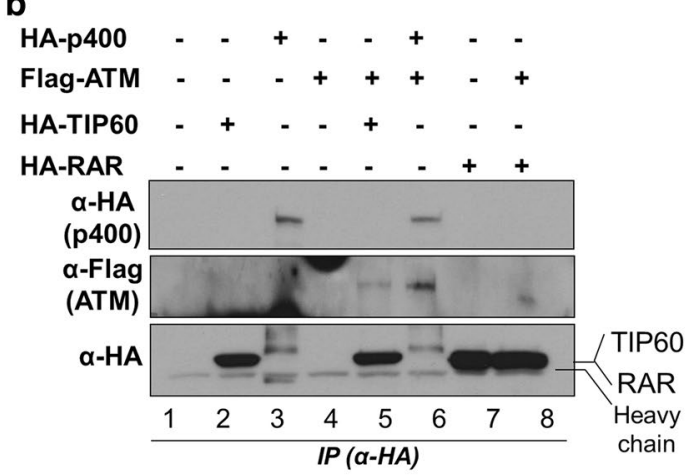

C

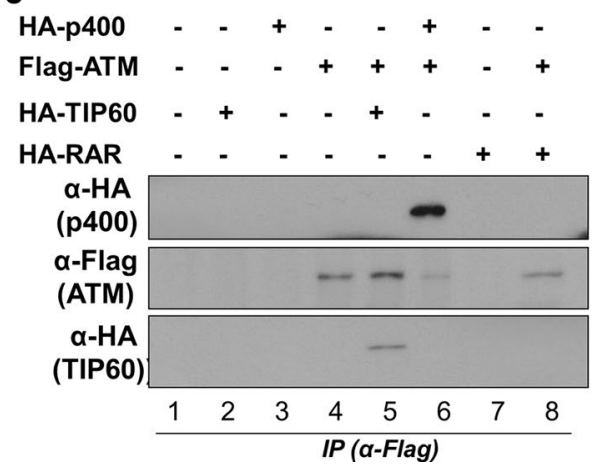

Fig. 2 Heterologous expression of ATM and p400 in Sf9 cells and their reciprocal interaction assays. a Cell lysate were prepared at $48 \mathrm{~h}$ post-infection and analysed by immunoblot or by Coomassie Brilliant Blue R-250 staining. b, c Equal volume of cell lysate from A was subjected to immunoprecipitation with anti-HA antibody conjugated beads (b) and anti-Flag antibody conjugated $\mathrm{M} 2$ agarose (c), respectively

Flag-ATM levels in lanes 5, 6). The interaction was further confirmed by performing the reciprocal immunoprecipitation using M2 agarose (Fig. 2c). The result reveals that HA-p400 co-purifies with ATM, similar to the result observed with HA-TIP60 (Fig. 2c, lanes 5, 6). Given that ATM and p400 were exclusively cytoplasmic in Sf9 cells for this interaction (Additional file 1: Figure $\mathrm{S} 1$ ), the possibility that both proteins bind to DNA and co-immunoprecipitate through indirect DNA bridges was excluded. Taken together, the reciprocal co-immunoprecipitation of ATM and p400 in Sf9 cells would suggest a protein-protein interaction which is comparable to or more efficient than the previously identified association of ATM and TIP60 [15].

\section{The N-terminal fragments of p400 interact with ATM}

To map the ATM interaction region on the $\mathrm{p} 400$ polypeptide, co-immunoprecipitation assays were performed using various p400 fragments expressed in HEK293T cells. A schematic representation of the p400 derivatives used in this mapping is shown in Fig. 3a. HA-ATM and Flag-p400 derivatives were transiently expressed in HEK293T cells and the whole cell extracts were subjected
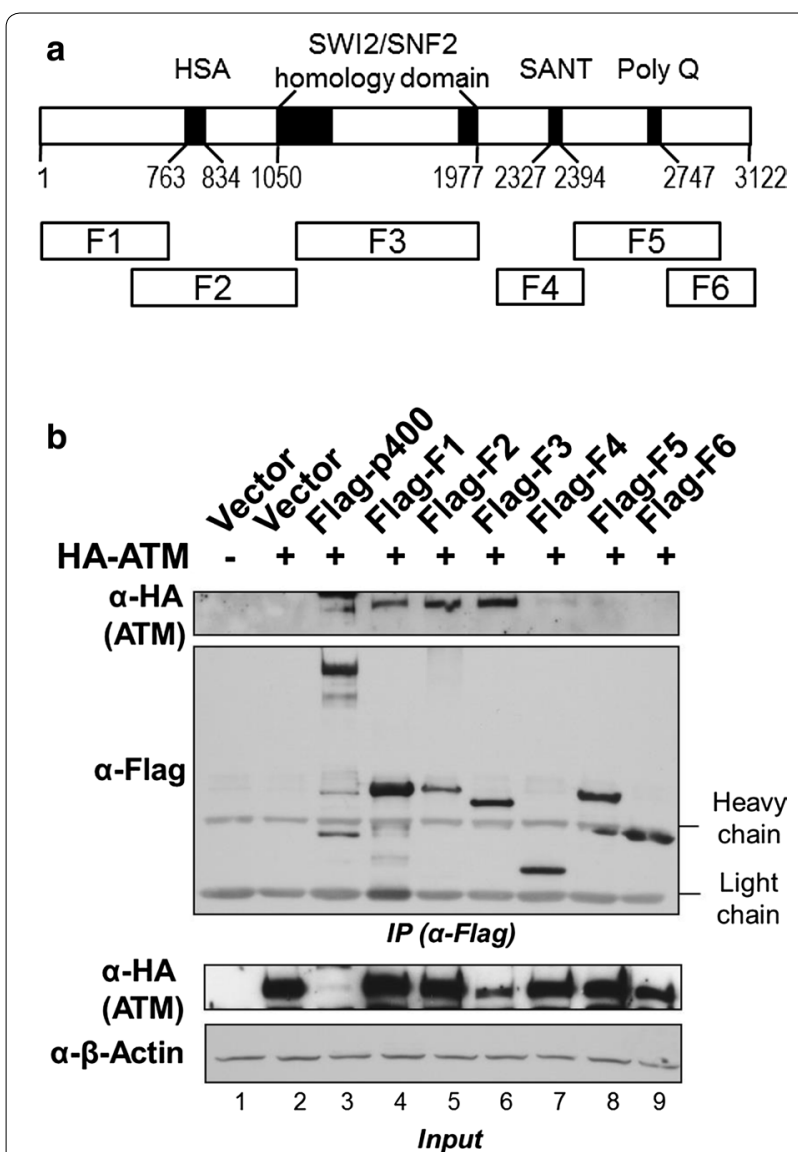

Fig. 3 ATM interaction domain mapping of p400. a Schematic presentation of the conserved domains on the p400 protein. HSA, Helicase/SANT-associated domain; SANT, SWI3-ADA2-N-CoR-TFIIIB domain. b Co-immunoprecipitation of ATM with fragment 1 (F1) to fragment 6 (F6) of p400. HEK293T cells were transfected with plasmids expressing HA-ATM alone or together with p400 and its derivatives 
to immunoprecipitation by M2 agarose (Fig. 3b). Ectopic HA-ATM expression levels varied among immunoprecipitated samples. Despite the effort to get similar levels of protein expression, ectopic ATM expression was barely detectable when co-expressed with full length Flag-p400 protein (Fig. 3b, input ATM panel, lane 3). To map the ATM interaction within $\mathrm{p} 400$, six sequential Flag-tagged fragments that spanned the $\mathrm{p} 400$ protein were used in co-immunoprecipitation experiments (Fig. 3b, IP panel, lanes 3-9). As expected, full length p400 co-immunoprecipitated with ATM but at a low level, which would result from the poor expression of ATM seen in the input sample (Fig. 3b, lane 3). The large band seen in lane 3 of both anti-HA panels is derived from residual staining of Flagp400 after insufficient stripping of the membrane prior to incubation with the anti-HA antibody. The three fragments spanning the N-terminal half of $\mathrm{p} 400$ were each able to associate with ATM (Fig. 3b, lanes 4-6). The association of multiple domains of p400 with ATM suggests that the interaction involves a direct protein interaction as well as indirect associations through bridging proteins. The F4 fragment of $\mathrm{p} 400$ was not associated with ATM but has been shown to interact with TIP60 previously, suggesting that the ATM-p400 interaction occurs in a manner distinct to that observed in the TIP60-p400 interaction [19].

\section{The expression of ATM-interacting fragments of $\mathrm{p} 400$ increases susceptibility to bleomycin without affecting ATM phosphorylation}

The depletion of $\mathrm{p} 400$ protein or forced overexpression of a catalytically-null p 400 revealed that $\mathrm{p} 400$ is required for decondensation of chromatin and subsequent recruitment of repair proteins [12, 17]. However, functional analysis of the ATM-p400 complex using siRNA mediated knockdown has been complicated as it does not differentiate a defective human NuA4 complex from a unique ATM-p400 association. The specific interaction between ATM and the N-terminal fragments of $\mathrm{p} 400$ suggests that it might provide a novel tool to study the DNA damage response. To this end, lentivirus was used to stably express the different $\mathrm{p} 400$ fragments in U2OS cells (Fig. 4, Additional file 2: Figure S2). To examine whether the expression of $\mathrm{p} 400$ fragments alters ATM activation and H2A.X phosphorylation after DNA damage, U2OS cells were exposed to the DNA damaging agent, bleomycin. The whole cell extracts were prepared at different post-recovery times after bleomycin treatment and the phosphorylation status of ATM and H2A.X was examined by immunoblotting (Fig. 4a). U2OS cells infected by control lentivirus (vector in Fig. 4a) showed a peak of ATM phosphorylation immediately after bleomycin treatment (Fig. 4a, lane 2) and returned to the basal level within a $3 \mathrm{~h}$ recovery period (Fig. 4a, P-ATM panel, compare lane 3 versus lane 1). Similar observations were made in each of the three U2OS cell lines expressing F1, F2 or F3 fragments. This result indicates that the ectopic expression of $\mathrm{p} 400$ fragments does not affect ATM autophosphorylation or the activation process. The clearance of ATM-mediated H2A.X phosphorylation is an indicator of how efficiently DNA damage is repaired. U2OS cells infected by control lentivirus (vector) showed a rapid clearance of $\gamma-\mathrm{H} 2 \mathrm{~A}$.X during a recovery period in which $\gamma$-H2A.X returned to a near basal level within $7 \mathrm{~h}$ (Fig. 4a, $\gamma$-H2A.X panel, compare lane 5 versus lane 1). U2OS cells expressing F3 displayed a similar pattern to the vector control whereas cells expressing either of $\mathrm{F} 1$ or F2 fragments showed a slower clearance of $\gamma$-H2A.X (Fig. 4b). These results indicate that the ectopic expression of ATM-interacting p400 fragments interferes with the clearance of $\gamma$-H2A.X and hinders DNA damage repair.

To determine if the ectopic expression of ATM-interacting p 400 fragments has any cytotoxic effect on normal cellular growth, cell numbers were monitored for 5 days in normal growth condition (Fig. 4c). U2OS cells expressing the F2 or F3 fragment showed a retarded growth compared to the control virus-infected cells. Given that the long-term stable expression levels of F2 and F3 fragments are much lower than that of transient transfection (Additional file 2: Figure S2A), cells with lower expression levels of these fragments appear to be preferentially selected for during the course of experiments. To examine whether the ectopic expression of an ATM-interacting p400 fragment affects drug susceptibility, the F1 and F6 fragments that did not inhibit cellular proliferation at a comparable expression level were tested for bleomycin susceptibility assay (Fig. $4 \mathrm{~d}$ and Additional file 2: Figure S2B). U2OS cells were exposed to bleomycin for $12 \mathrm{~h}$ and the cellular growth was monitored for 7 days. While the cells infected with virus for vector control or F6 fragment showed a comparable recovery and growth, the ectopic expression of ATM-interacting F1 fragment resulted in severely restricted growth during the post-recovery period.

To investigate how the ectopic expression of F1 fragment distinctively affects the ATM-p400 interaction from the TRRAP-p400 complex, HEK293T cell line stably expressing Flag-F1 was generated and used to compare the amount of co-immunoprecipitated ATM and TRRAP with $\mathrm{p} 400$, respectively. Transient expression of HA-p400 was performed in duplicate in HEK293T cell lines (Fig. 5a, lanes 1-12). The cell lysates were precleared by protein A/G beads and subjected to immunoprecipitation using anti-HA antibody-conjugated beads. As expected, the amount of TRRAP was significantly 


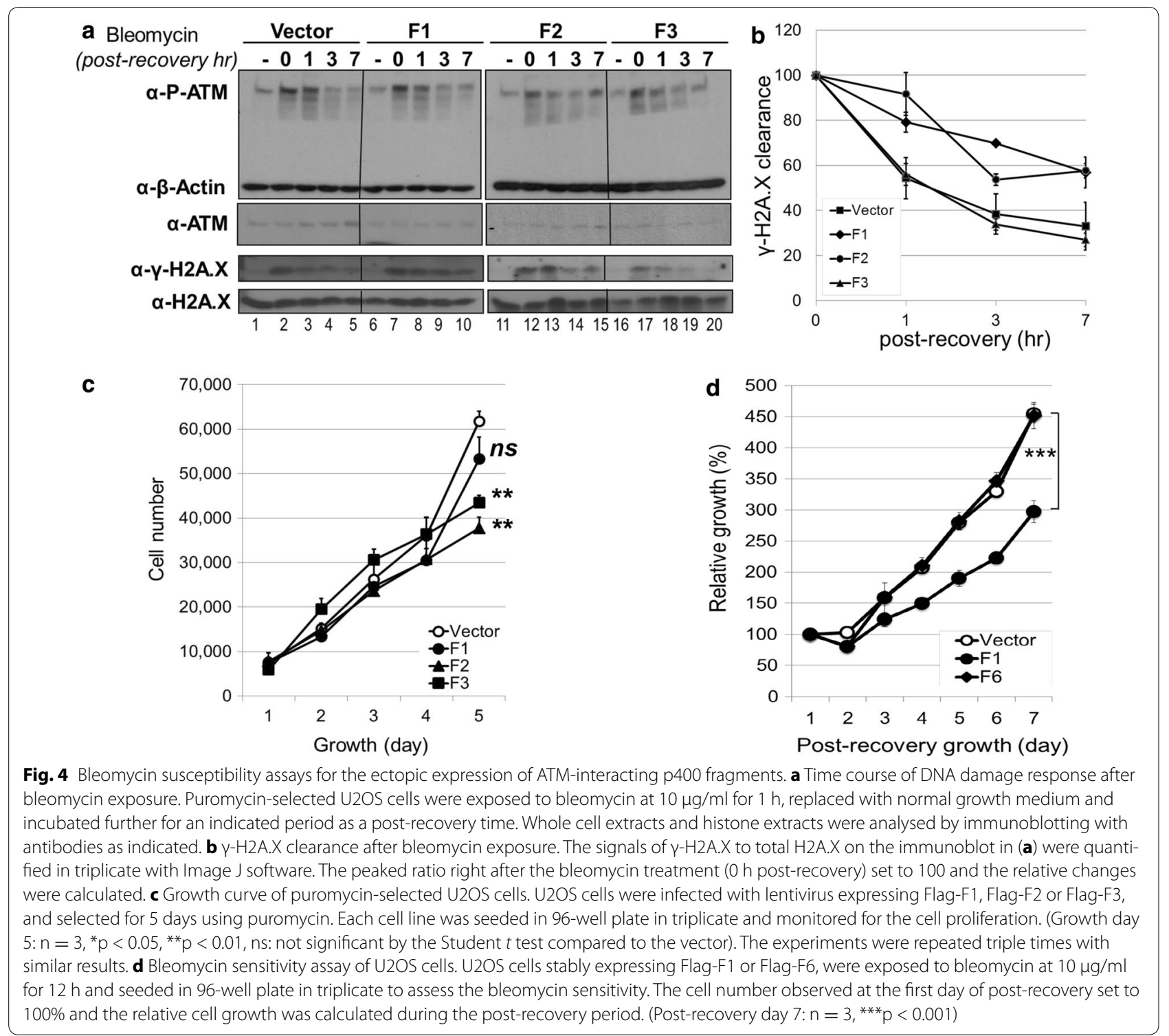

enriched by HA-p400 immunoprecipitation but was not altered by F1 fragment expression (Fig. 5a, lanes 5-6 and $11-12$ of the $\alpha$-TRRAP panel). However, the amount of p400-associated ATM was consistently reduced in the presence of F1 expression (Fig. 5a, lanes 5-6 and 11-12 of the $\alpha$-ATM panel), suggesting that F1 interferes with the protein-protein interaction between ATM and p400.

In addition to the strong association with ATM in our studies, the F2 and F3 fragments covering helicase/SANT-associated (HSA) and SWI/SNF2 homology domains have previously been shown to interact with various subunits of $\mathrm{NuA} 4$ complex and serve as a critical core for the complex assembly $[20,26]$. Therefore, similar interference studies were done to investigate whether the overexpressed F2 or F3 affects the
ATM-p400 interaction (Fig. 5b). The result shows that F3 fragment, albeit its minimal expression level, significantly reduced the amount of ATM associated with p400, suggesting that the SWI/SNF2 homology domain of p 400 would also specifically interfere with the protein-protein interactions of ATM-p400 (Fig. 5b, lane 6 of anti-ATM immunoblot). In contrast, neither of the fragments affects the amount of co-immunoprecipitated TRRAP by HA-p400 (Fig. 5b, lanes $4-6$ of anti-TRRAP immunoblot). Interestingly, both F2 and F3 but not F1 were co-immunoprecipitated with HA-p400, implying that various NuA4 subunits that interact with F2 or F3 might play a role as a bridging protein to bring a fulllength p400 together with its fragments (Fig. 5b, lanes 5-6 of anti-Flag immunoblot). 

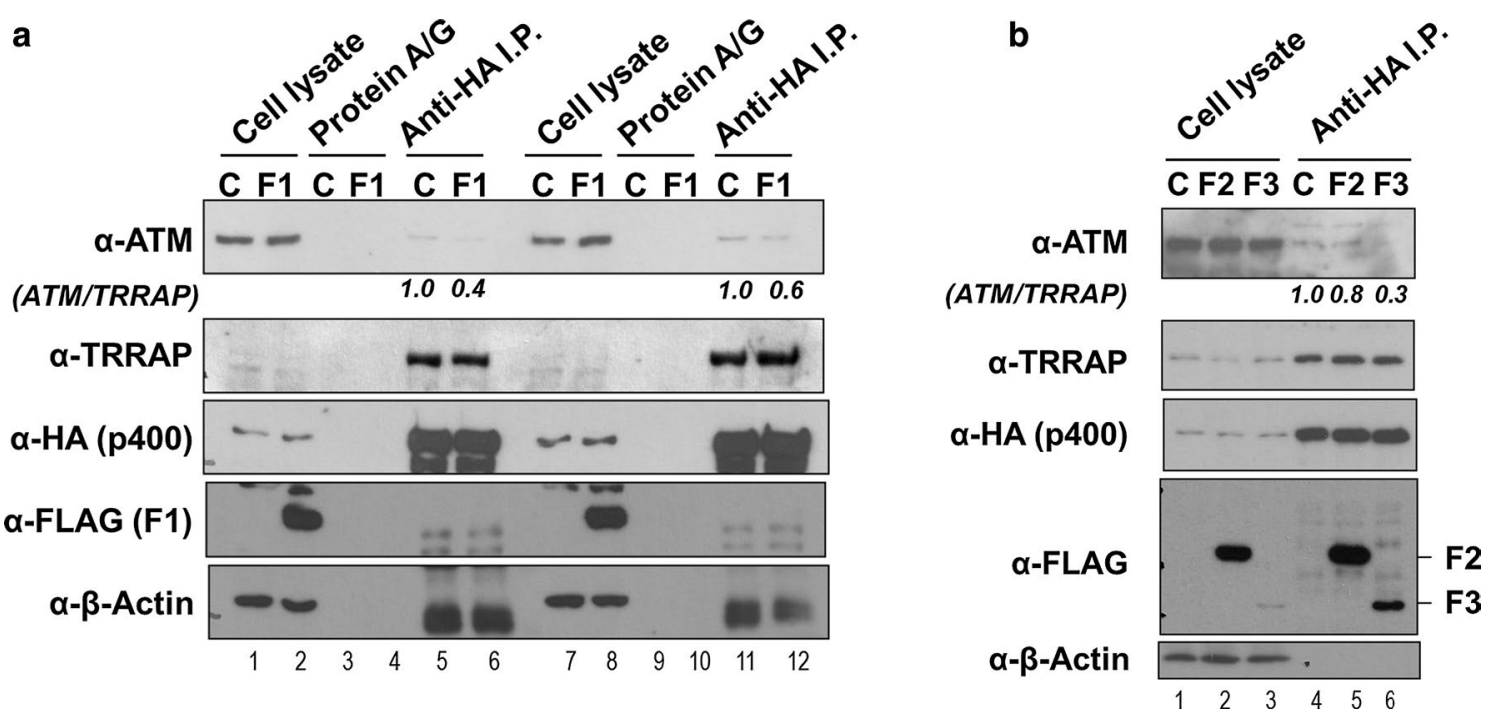

Fig. 5 The N-terminal fragments of p400 interfere with the protein-protein interaction between ATM and p400. a HEK293T cells virally transduced by either control or F1 fragment expressing lentivirus were selected against puromycin and then used for the transient expression of HA-p400 in duplicate. The cell lysates were pre-cleared by protein A/G beads for $3 \mathrm{~h}$ and subjected to immunoprecipitation by anti-HA antibody-conjugated beads. Two percent of prepared cell lysates was used as input to ensure comparable expression levels of ATM, TRRAP and p400 in control (C) and Flag-F1 fragment (F1) cell lines. The immunoblot signals from co-immunoprecipitated ATM and TRRAP were quantified using Image I software and the ratios of ATM to TRRAP were calculated (ATM/TRRAP). b The same experiments were conducted except the transient expression of F2 and F3 fragments in HEK293T cells. The data represents one of two independent experiments with similar results

Taken together, ectopic expression of the N-terminal fragments of p400 lead to more severe drug-induced cytotoxicity or retarded cellular growth, and the underlying mechanism might involve the interference of $\mathrm{p} 400$ function with ATM or human NuA4 complexes.

\section{Discussion}

Here we report that a catalytic subunit of the TRRAPTIP60 complex, p400 can associate with ATM in both HEK293T and Sf9 cells. The association between ATM and p400 is not surprising since FATC domains of ATM and TRRAP are functionally exchangeable for interaction partners such as TIP60 [18]. The MRN (Mre11-Rad50$\mathrm{Nbs1)}$ complex has also been shown to interact physically and functionally with both ATM and TRRAP during DSB end joining processes [27]. The protein-protein interaction between ATM and p400 appears to occur independently of DNA damage and the interacting p400 complex would be unique since the integral subunits of TRRAPTIP60 complexes, BAF53 and GAS41 did not associate with ATM. Despite the reciprocal interaction of ATM and p400 in Sf9 cells, we cannot exclude a possibility that the association of p400 with ATM is mediated by indirect associations through unknown insect bridging proteins. It is important to note that in this study the interaction between ATM and p400 was seen mainly through the use of overexpressed proteins, although Flag-tagged p400 was able to precipitate endogenous ATM (Fig. 1b). The failure to co-immunoprecipitate the two proteins endogenously is consistent with a recent analysis of endogenous p400 and ATM complexes that did not show the stoichiometric association of p400 or TIP60 [28]. Therefore, it is possible that the interaction between ATM and p400 seen in this study may represent a transient interaction only present in a certain cellular condition or derived from a minor fraction of the endogenous complexes.

Suppression of the ATM-p400 interaction appears to inhibit DSB repair as shown through our experiments using stably expressed p400 fragments in U2OS cells. However, clear functional roles in the DNA repair process remain to be elucidated. We speculate that the ATM-p400 association could influence the DNA damage response in two different ways either regulating ATM activity or targeting the p400 protein to the DSB site. Firstly, the p400 protein could regulate the activity of ATM or ATM-associated TIP60. In support of this, p400 is known to be a negative regulator of TIP60 when they are within the same TRRAP-TIP60 complex [19]. Previously, ATM activation has been shown to be more robust and rapid in p400-depleted cells, suggesting that $\mathrm{p} 400$ is a negative regulator of ATM activation [17]. More importantly, we show that the interaction between ATM and p400 is not altered regardless of DNA damage, suggesting that the inhibitory effects on ATM would be regulated by 
post-translational modifications [29]. As a second functional role, ATM may serve to ferry p400 to locations of DNA damage. This speculation would explain a previous observation that the loss of ATM reduces or delays histone loss at the DSB site [30]. The association of ATMp400 may allow a rapid recruitment of p400 activity to the DNA double-strand break site as ATM is one of the earliest DNA repair proteins found at the DNA damage sites [31]. By increasing the local concentration of p400 together with TRRAP and TIP60 at the DNA break site, dynamic complex assembly would be achieved for efficient chromatin remodeling activity [12].

Our results show that N-terminal fragments of $\mathrm{p} 400$ interact with ATM in a similar but distinct manner to that observed in the p400-TIP60 interaction. In particular, stable expression of $\mathrm{p} 400$ fragments containing the helicase-SANT-associated (HSA) domain (F2) or the SWI/SNF homology domain (F3) in U2OS cells are inhibitory to the cellular growth. The F2 and F3 regions of 4400 have been shown to be important in the integrity and assembly of the TRRAP-TIP60 complex [20, 26, 32]. Given that the functional loss of TRRAP induces cell proliferation arrest [33], it is intriguing to know whether the F2 or F3 region of $\mathrm{p} 400$ would directly interfere with the function of TRRAP-TIP60 complexes and cause a growth inhibitory effect. On the other hand, the stable expression of the N-terminal F1 fragment (1-719) of p400 did not interfere with normal cellular growth but sensitised the cells upon bleomycin treatment. F1 does not have any conserved domain that has been previously shown to interact with other chromatin remodeling complexes or DNA repair proteins. Therefore, the F1 fragment-induced sensitisation in U2OS cells is unlikely to be due to the inhibition of the TRRAP-TIP60 complex or changes in gene expression, but more likely caused by interfering with the interaction between ATM and p400 as shown in Fig. 5a. A dominant negative approach using the F1 fragment will be useful to further dissect the complicated functions carried out by multiple p 400 complexes.

\section{Conclusion}

ATM interacts with p400 independently of DNA damage in HEK293T cells and their interaction may represent a transient minor interaction under a certain physiological condition. The N-terminal domains of $\mathrm{p} 400$ is vital for the interaction with ATM. Based on these findings, we have shown that the expression of the $\mathrm{N}$-terminal $\mathrm{p} 400$ fragment sensitizes U2OS cells to bleomycin.

\section{Methods}

\section{Cell culture}

HEK293T and U2OS cells were originally obtained from ATCC (Rockville, MD). Cells were routinely maintained on a regular basis in the presence of mycoplasma preventive antibiotics, plasmocin $(5 \mu \mathrm{g} / \mathrm{ml}$, Invivogen $)$ and confirmed for the absence of mycoplasma contamination. HEK293T and U2OS cells were maintained in Dulbecco's modified Eagle's medium (Gibco/Life Technology) containing $10 \%$ fetal calf serum (FCS, Sigma Aldrich) and supplemented with $50 \mathrm{U} / \mathrm{ml}$ penicillin, $50 \mu \mathrm{g} / \mathrm{ml}$ streptomycin and $0.125 \mu \mathrm{g} / \mathrm{ml}$ amphotericin B (Sigma Aldrich) and cultured at $37{ }^{\circ} \mathrm{C}$ in a $5 \% \mathrm{CO}_{2}$ humidified incubator. Sf9 cells were cultured in normal atmospheric conditions in supplemented Grace's Insect Media (Gibco/Life Technology) with 10\% FCS (Sigma), 0.1\% Pluronic acid (Sigma), $10 \mu \mathrm{g} / \mathrm{ml}$ gentamycin, $50 \mathrm{U} / \mathrm{ml}$ penicillin, $50 \mu \mathrm{g} /$ $\mathrm{ml}$ streptomycin and $0.125 \mu \mathrm{g} / \mathrm{ml}$ amphotericin B (Sigma Aldrich). Baculovirus and lentivirus were prepared using methods described in the manufacturer's procedure (Invitrogen and System Biosciences, respectively). For the analysis of cellular proliferation, the Cyquant cell proliferation assay kit (Invitrogen, C7026) was used as described previously [34].

\section{Plasmid and antibodies}

The cDNA of Flag-tagged ATM was obtained from Dr Chris Bakkenist (University of Pittsburg, USA). Mammalian expression vectors for $\mathrm{p} 400$, $\mathrm{p} 400$-derived fragments (F1-F6), TIP60, BAF53 and GAS41 were described in previous studies $[19,23,35,36]$. All other plasmids in this study including baculoviral and lentiviral expression vectors were constructed by standard cloning procedures. The primary antibodies used in this study were H2AX (Milipore, 07-627), H2AX S139P (Milipore, 07-164), $\beta$-actin (Sigma, A5316), ATM (Calbiochem, 819844), P-ATM (Serine 1981, Abcam, ab36810), Flag M2 (Sigma, F3165), and HA (Sigma, SAB4300603). Antibodies against TIP60 and p400 were produced from rabbits and have been used in previous studies.

\section{Protein extraction, immunoprecipitation and immunoblotting}

HEK293T and Sf9 cells were harvested by scraping into PBS, pelleted and resuspended in lysis buffer $(20 \mathrm{mM}$ HEPES pH 7.4, $120 \mathrm{mM} \mathrm{NaCl}, 1.5 \mathrm{mM} \mathrm{MgCl}, 1 \mathrm{mM}$ EGTA, $50 \mathrm{mM}$ NaF, $0.2 \%$ Tween-20, $0.5 \mathrm{mM}$ DTT, $1 \mathrm{mM}$ PMSF). The lysed supernatant was used as whole cell extracts. To extract histones from the insoluble fraction, the pellet after soluble protein extraction was resuspended in $1 \mathrm{ml} \mathrm{H}_{2} \mathrm{O}$ then centrifuged at $16,000 \mathrm{~g}$ for $10 \mathrm{~min}$. The supernatant was aspirated and the pellet was resuspended in $200 \mu \mathrm{l}$ of $0.2 \mathrm{~N} \mathrm{HCl}$ and left on ice overnight. The sample was centrifuged at $16,000 \mathrm{~g}$ for $10 \mathrm{~min}$ and the supernatant was transferred to a new microfuge tube before being neutralised with $10 \mu \mathrm{l}$ of 2 M Tris, pH 10. Anti-Flag (M2) affinity gel (Sigma) or 
EZview $^{\text {TM }}$ Red Anti-HA affinity gel (Sigma) were added to the cell lysate and incubated overnight at $4{ }^{\circ} \mathrm{C}$. Beads were washed and analysed by an SDS-PAGE on a $5-10 \%$ two step gradient gel followed by the immunoblot procedure.

\section{Additional files}

Additional file 1: Figure S1. Sf9 cells were infected with the baculovirus expressing indicated heterologous transgenes and cultured for 24, 36 or $48 \mathrm{~h}$ before processing. (A) Flag-ATM (red) is found in the nucleus (identified by DAPI staining; blue) as well as in the cytosplasm (cell boundaries are visible in the DIC image) at all-time points. (B) HA-p400 (green) is also distributed in all cellular compartments $24 \mathrm{~h}$ after infection. As time advances $(36,48 \mathrm{~h}$ ), the protein exits from the nucleus (delineated by DAPI; blue) until it is exclusively cytoplasmic. (C) cells co-expressing FlagATM (red) and HA-p400 (green) exhibit dynamic protein relocalisation. $24 \mathrm{~h}$ post-infection a common pool of ATM and p400 is observed in the nucleus (DAPI; blue). The nuclear localisation of both proteins decreases with a concomitant enrichment of the cytoplasmic fraction $(36,48 \mathrm{~h})$. All scale bars are $5 \mu \mathrm{m}$.

Additional file 2: Figure S2. (A) ectopic expression of ATM-interacting p400 fragments in U2OS cells. U2OS cells were infected with lentivirus expressing Flag-tagged p400 fragments and selected for 5 days against puromycin. Total $500 \mu \mathrm{g}$ of protein was immunoprecipitated with M2 agarose and analysed by immunoblotting with anti-Flag antibody. (B) growth curve of puromycin-selected U2OS cells. U2OS cells were infected with lentivirus expressing Flag-F1or Flag-F6, and selected for 5 days against puromycin. Each cell line was seeded in 96-well plate in triplicate and monitored for the cell proliferation.

\section{Abbreviations}

ATM: ataxia telangiectasia mutated; TRRAP: transformation/transcription domain associated protein; PIKK: phosphoinositide 3-Kinase-related protein kinase; DSB: DNA double-strand break; NuA4: nucleosome acetyltransferase of H4; FATC: FRAP-ATM-TRRAP-C-terminal; HEK293T: human embryonic kidney 293T; RAR: retinoic acid receptor; HSA: Helicase/SANT-Associated; SANT: SWI/ SNF2, Ada, N-CoR, TFIIIB; SWI/SNF2: SWItch, sucrose non-fermentable; MRN: Mre11-Rad50-Nbs1; FCS: fetal calf serum; PBS: phosphate-buffered saline; HEPES: 4-(2-hydroxyethyl)-1-piperazineethanesulfonic acid; EGTA: ethylene glycol tetraacetic acid; DTT: Dithiothreitol; PMSF: phenylmethane sulfonyl fluoride; SDS-PAGE: sodium dodecyl sulfate polyacrylamide gel electrophoresis; DAPI: 4',6-Diamidino-2-Phenylindole.

\section{Authors' contributions}

RJS conducted the experiments, analysed the results, and wrote some of the paper. MSS conducted experiments for the protein localisation studies in the insect cells with RJS. LEW conducted the experiments for the lentiviral expression of the $\mathrm{p} 400$ fragments with JHP. JHP conceived the idea of the project, conducted the experiments, analysed the results, and wrote the paper. All authors read and approved the final manuscript.

\section{Acknowledgements}

We thank Dr. Kathryn Stowell and Rachel Miller for critical reading of this manuscript. We further acknowledge Dr Fata Moradali for technical assistance and the Manawatu Microscopy and Imaging Centre for scanning confocal microscopy use.

\section{Competing interests}

The authors declare that they have no competing interests.

\section{Availability of data and materials}

All data from this study are contained within the manuscript or the supplemental data.

\section{Ethics approval and consent to participate}

No human or animal subjects were used in this study. Declarations of ethical treatment of human subjects or animals, or consent of human subjects are therefore not applicable.

\section{Funding}

This work was supported by a grant from Massey University Research Fund, Genesis Oncology Trust, and Palmerston North Medical Research Foundation to J.H.P and New Zealand Federation of Graduate Women to RJS. Neither of funding bodies has participated in the design of the study and collection, analysis, and interpretation of data.

Received: 19 December 2015 Accepted: 29 October 2016

Published online: 04 November 2016

\section{References}

1. Lempiainen $\mathrm{H}$, Halazonetis TD. Emerging common themes in regulation of PIKKs and PI3Ks. EMBO J. 2009;28(20):3067-73.

2. Murr R, Loizou Jl, Yang YG, Cuenin C, Li H, Wang ZQ, Herceg Z. Histone acetylation by Trrap-Tip60 modulates loading of repair proteins and repair of DNA double-strand breaks. Nat Cell Biol. 2006;8(1):91-9.

3. Burma S, Chen BP, Murphy M, Kurimasa A, Chen DJ. ATM phosphorylates histone H2AX in response to DNA double-strand breaks. J Biol Chem. 2001;276(45):42462-7.

4. Falck J, Coates J, Jackson SP. Conserved modes of recruitment of ATM, ATR and DNA-PKcs to sites of DNA damage. Nature. 2005:434(7033):605-11.

5. Matsuoka S, Ballif BA, Smogorzewska A, McDonald ER 3rd, Hurov KE, Luo J, Bakalarski CE, Zhao Z, Solimini N, Lerenthal Y, et al. ATM and ATR substrate analysis reveals extensive protein networks responsive to DNA damage. Science. 2007;316(5828):1160-6.

6. Canman CE, Lim DS, Cimprich KA, Taya Y, Tamai K, Sakaguchi K, Appella E, Kastan MB, Siliciano JD. Activation of the ATM kinase by ionizing radiation and phosphorylation of p53. Science. 1998;281(5383):1677-9.

7. Xu B, Kim ST, Lim DS, Kastan MB. Two molecularly distinct $G(2) / M$ checkpoints are induced by ionizing irradiation. Mol Cell Biol. 2002;22(4):1049-59

8. Geng L, Zhang $X$, Zheng S, Legerski RJ. Artemis links ATM to G2/M checkpoint recovery via regulation of Cdk1-cyclin B. Mol Cell Biol. 2007;27(7):2625-35.

9. Gatei M, Jakob B, Chen P, Kijas AW, Becherel OJ, Gueven N, Birrell G, Lee $J H$, Paull TT, Lerenthal Y, et al. ATM protein-dependent phosphorylation of Rad50 protein regulates DNA repair and cell cycle control. J Biol Chem. 2011;286(36):31542-56.

10. Ziv Y, Bielopolski D, Galanty Y, Lukas C, Taya Y, Schultz DC, Lukas J, BekkerJensen S, Bartek J, Shiloh Y. Chromatin relaxation in response to DNA double-strand breaks is modulated by a novel ATM- and KAP-1 dependent pathway. Nat Cell Biol. 2006;8(8):870-6.

11. Lee JH, Paull TT. ATM activation by DNA double-strand breaks through the Mre11-Rad50-Nbs1 complex. Science. 2005;308(5721):551-4.

12. $X u Y$, , Sun $Y$, Jiang $X$, Ayrapetov MK, Moskwa $P$, Yang $S$, Weinstock DM, Price BD. The p400 ATPase regulates nucleosome stability and chromatin ubiquitination during DNA repair. J Cell Biol. 2010;191 (1):31-43.

13. Kruhlak MJ, Celeste A, Dellaire G, Fernandez-Capetillo O, Muller WG, McNally JG, Bazett-Jones DP, Nussenzweig A. Changes in chromatin structure and mobility in living cells at sites of DNA double-strand breaks. J Cell Biol. 2006;172(6):823-34.

14. Doyon Y, Cote J. The highly conserved and multifunctional NuA4 HAT complex. Curr Opin Genet Dev. 2004;14(2):147-54.

15. Squatrito M, Gorrini C, Amati B. Tip60 in DNA damage response and growth control: many tricks in one HAT. Trends Cell Biol. 2006;16(9):433-42.

16. Sun Y, Jiang X, Price BD. Tip60: connecting chromatin to DNA damage signaling. Cell Cycle. 2010;9(5):930-6.

17. Courilleau C, Chailleux C, Jauneau A, Grimal F, Briois S, Boutet-Robinet $E_{\text {, }}$ Boudsoca F, Trouche D, Canitrot Y. The chromatin remodeler p400 ATPase facilitates Rad51-mediated repair of DNA double-strand breaks. J Cell Biol. 2012;199(7):1067-81. 
18. Jiang X, Sun Y, Chen S, Roy K, Price BD. The FATC domains of PIKK proteins are functionally equivalent and participate in the Tip60-dependent activation of DNA-PKcs and ATM. J Biol Chem. 2006;281(23):15741-6.

19. Park JH, Sun XJ, Roeder RG. The SANT domain of p400 ATPase represses acetyltransferase activity and coactivator function of TIP60 in basal p21 gene expression. Mol Cell Biol. 2010;30(11):2750-61.

20. Fuchs M, Gerber J, Drapkin R, Sif S, Ikura T, Ogryzko V, Lane WS, Nakatan Y, Livingston DM. The p400 complex is an essential E1A transformation target. Cell. 2001;106(3):297-307.

21. Sun $Y$, Jiang $X$, Chen S, Fernandes N, Price BD. A role for the Tip60 histone acetyltransferase in the acetylation and activation of ATM. Proc Natl Acad Sci USA. 2005;102(37):13182-7.

22. Cai Y, Jin J, Florens L, Swanson SK, Kusch T, Li B, Workman JL, Washburn MP, Conaway RC, Conaway JW. The mammalian YL1 protein is a shared subunit of the TRRAP/TIP60 histone acetyltransferase and SRCAP complexes. J Biol Chem. 2005;280(14):13665-70.

23. Park JH, Roeder RG. GAS41 is required for repression of the $p 53$ tumor suppressor pathway during normal cellular proliferation. Mol Cell Biol. 2006;26(11):4006-16.

24. Kusch T, Florens L, Macdonald WH, Swanson SK, Glaser RL, Yates JR, Abmayr SM, Washburn MP, Workman JL. Acetylation by Tip60 is required for selective histone variant exchange at DNA lesions. Science (New York, NY). 2004;306(5704):2084-7.

25. Sun $Y, X u Y$, Roy $K$, Price BD. DNA damage-induced acetylation of lysine 3016 of ATM activates ATM kinase activity. Mol Cell Biol. 2007;27(24):8502-9.

26. Auger A, Galarneau L, Altaf M, Nourani A, Doyon Y, Utley RT, Cronier D, Allard S, Cote J. Eaf1 is the platform for NuA4 molecular assembly that evolutionarily links chromatin acetylation to ATP-dependent exchange of histone H2A variants. Mol Cell Biol. 2008;28(7):2257-70.
27. Robert F, Hardy S, Nagy Z, Baldeyron C, Murr R, Dery U, Masson JY, Papadopoulo D, Herceg Z, Tora L. The transcriptional histone acetyltransferase cofactor TRRAP associates with the MRN repair complex and plays a role in DNA double-strand break repair. Mol Cell Biol. 2006;26(2):402-12.

28. Dalvai M, Loehr J, Jacquet K, Huard CC, Roques C, Herst P, Cote J, Doyon $Y$. A scalable genome-editing-based approach for mapping multiprotein complexes in human cells. Cell Rep. 2015;13(3):621-33.

29. Stokes MP, Rush J, Macneill J, Ren JM, Sprott K, Nardone J, Yang V, Beausoleil SA, Gygi SP, Livingstone M, et al. Profiling of UV-induced ATM/ATR signaling pathways. Proc Natl Acad Sci USA. 2007;104(50):19855-60.

30. Berkovich E, Monnat RJ Jr, Kastan MB. Roles of ATM and NBS1 in chromatin structure modulation and DNA double-strand break repair. Nat Cell Biol. 2007;9(6):683-90.

31. Uziel T, Lerenthal $Y$, Moyal $L$, Andegeko Y, Mittelman L, Shiloh $Y$. Requirement of the MRN complex for ATM activation by DNA damage. EMBO J. 2003;22(20):5612-21.

32. Jha S, Gupta A, Dar A, Dutta A. RVBs are required for assembling a functional TIP60 complex. Mol Cell Biol. 2013;33(6):1164-74.

33. Herceg Z, Hulla W, Gell D, Cuenin C, Lleonart M, Jackson S, Wang ZQ. Disruption of Trrap causes early embryonic lethality and defects in cell cycle progression. Nat Genet. 2001;29(2):206-11.

34. Park JH, Hale TK, Smith RJ, Yang T. PPM1B depletion induces premature senescence in human IMR-90 fibroblasts. Mech Ageing Dev. 2014; 138:45-52.

35. Park J, Wood MA, Cole MD. BAF53 forms distinct nuclear complexes and functions as a critical c-Myc-interacting nuclear cofactor for oncogenic transformation. Mol Cell Biol. 2002:22(5):1307-16.

36. Lee K, Lau ZZ, Meredith C, Park JH. Decrease of p400 ATPase complex and loss of H2A.Z within the p21 promoter occur in senescent IMR-90 human fibroblasts. Mech Ageing Dev. 2012;133(11-12):686-94.

\section{Submit your next manuscript to BioMed Central and we will help you at every step:}

- We accept pre-submission inquiries

- Our selector tool helps you to find the most relevant journal

- We provide round the clock customer support

- Convenient online submission

- Thorough peer review

- Inclusion in PubMed and all major indexing services

- Maximum visibility for your research

Submit your manuscript at www.biomedcentral com/submit 